\title{
Controlled/“Living” Radical Ring-Opening Polymerization of 5,6-Benzo-2-Methylene-1,3-Dioxepane Based on Reversible Addition-Fragmentation Chain Transfer Mechanism
}

\author{
Tao He, Ying-Fang Zou, and Cai-Yuan PAN ${ }^{\dagger}$ \\ Department of Polymer Science and Engineering, University of Science and \\ Technology of China, Hefei, Anhui 230026, Peoples Republic of China
}

(Received September 17, 2001; Accepted December 28, 2001)

\begin{abstract}
The radical ring-opening polymerization of 5,6-benzo-2-methylene-1,3-dioxepane (BMDO) was performed in the presence of 1-(ethoxycarbonyl)prop-1-yl-dithiobenzoate (EPDTB) and dicumyl peroxide (DCP). The completely ring-opening polymerization of BMDO via reversible addition-fragmentation transfer mechanism was proved to be controlled by the following experimental evidences: the straight line of $\ln \left([M]_{0} /[M]_{\mathrm{t}}\right) v s$. polymerization time; linear increase of molecular weight with increasing conversion and relatively narrow molecular weight distribution. The mechanism of the polymerization was discussed. The complete ring-opened structure of the PBMDO was characterized by ${ }^{1} \mathrm{H}$ and ${ }^{13} \mathrm{C}$ NMR spectroscopies as well as gel permeation chromatography (GPC).

KEY WORDS Controlled Polymerization / Radical Ring-Opening Polymerization / Reversible Addition-Fragmentation Chain Transfer (RAFT) / 5,6-Benzo-2-Methylene-1,3-Dioxepane /
\end{abstract}

Free radical ring-opening polymerization has been an attractive subject because it produces polymers with various functional groups (such as ethers, esters, amides, and carbonates) in their backbone. ${ }^{1-14}$ Addition of unsaturated heterocycles into polymerization system of commercial monomers will improve the properties of the polymers obtained. For example, when 2-methylene-4- $n$-decyl-1,3-dioxolane was copolymerized with vinyl chloride, the thermal stability and flexibility of the polymer obtained improved. ${ }^{15}$ However, free radical ring-opening polymerization of the unsaturated heterocycles yields polymers with low molecular weight and broad molecular weight distribution. For instance, the radical ring-opening polymerization of 2-methylene-4-phenyl-1,3-dioxolane proceeds via chain radicals 1 and 2 as shown in Scheme $1 .^{16-18}$ The chain radicals 1 and 2 are stabilized respectively by two oxygens and phenyl group. The former radical is more active than the later one. The low molecular weight polymer with broad polydispersity index may be due to the transfer reaction during the polymerization.

Recently, significant progress has been made in the field of living free radical polymerization including nitroxide-mediate stable free radical polymerization (SFRP) $;{ }^{19-23}$ atom transfer radical polymerization (ATRP), ${ }^{24-26}$ and reversible addition-fragmentation transfer (RAFT) process. ${ }^{27,} 28$ In these polymerizations, there is a fast equilibrium between propagating radi-

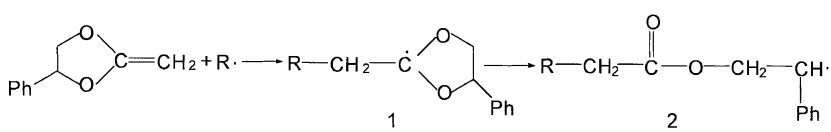

Scheme 1.

cals and dormant chains, which is prerequisite for the synthesis of controlled polymers. ${ }^{29}$ When the methods apply in the polymerization of unsaturated heterocycles, the termination of propagating radicals must be affected. To produce polymers with controlled molecular weights and architectures, Wei et al. ${ }^{30}$ reported the study on nitroxide-mediated stable free radical polymerization of 2-methylene-1,3-dioxepane, which underwent quantitative ring-opening polymerization to afford a polyester with polydispersity less than 1.5. Jia et $a l^{31}$ presented the first example of a controlled free radical double ring-opening polymerization of 8,9benzo-2-methylene-1,4,6-trioxaspiro[4,4]nonane. In our research program on controlled free radical ringopening polymerization of unsaturated cyclic acetals, cyclic ethers and spiro orthocarbonates, we studied controlled radical ring-opening polymerization of several unsaturated heterocycles and block copolymerization of these cyclic compounds with some commercial monomers. In the case of 2-methylene-4-phenyl1,3-dioxolane (MPDO), the ATRP of MPDO yielded a polymer consisted of addition and ring-opening units with well-controlled molecular weight and narrow molecular weight distribution, ${ }^{32}$ which is different from its conventional free radical polymerization. ${ }^{2}$ How-

${ }^{\dagger}$ To whom all correspondence should be addressed (E-mail: pcy@ustc.edu.cn). 
ever, 5,6-benzo-2-methylene-1,3-dioxepane (BMDO) underwent complete ring-opening polymerization using ATRP initiator system, a polyester with wellcontrolled molecular weight and narrow polydispersity was produced. ${ }^{33}$ We want to clarify whether RAFT process can be extended to free radical ring-opening polymerization of unsaturated cyclic acetals. Here, we report the controlled radical ring-opening polymerization of BMDO with 1-(ethoxycarbonyl)prop-1-yldithiobenzoate (EPDTB) and dicumyl peroxide (DCP) as transfer agent and initiator respectively.

\section{EXPERIMENTAL}

\section{Materials}

The monomer BMDO was synthesized according to the method described in ref $1,{ }^{1} \mathrm{H}$ NMR $(500 \mathrm{MHz}$, $\mathrm{CDCl}_{3}$ ), $\delta$ (TMS, ppm), 7.05-7.3 (m, $4 \mathbf{H}$, aromatic $\mathrm{Hs}$ ), 5.06 (s, 4H, 2OCH $\left.\boldsymbol{C H}_{2}\right), 3.70$ (s, 2H, $\boldsymbol{C H}_{2}=\mathrm{C}$ ).

Dicumyl peroxide (DCP analytical grade) was purchased from Shanghai Chemical Reagent Plant and used as received. All other reagents used were of analytical grade.

\section{Synthesis of EPDTB}

The transfer agent EPDTB was prepared as follows: Magnesium ( $2 \mathrm{~g}, 0.083 \mathrm{~mol})$ and $80 \mathrm{~mL}$ dry THF were added into a $250 \mathrm{~mL}$ three-necked flask, and then, $15.7 \mathrm{~g}(0.1 \mathrm{~mol})$ of bromobenzene was dropwise added into the reaction mixture in $0.5 \mathrm{~h}$. The reaction was carried out at $40^{\circ} \mathrm{C}$ until magnetism was completely disappeared. Then, carbon disulfide $(7.62 \mathrm{~g}, 0.1 \mathrm{~mol})$ was dropped in about $20 \mathrm{~min}$. After reaction proceeded for $4 \mathrm{~h}$ at $40^{\circ} \mathrm{C} 17.5 \mathrm{~g}(0.09 \mathrm{~mol})$ ethyl $\alpha$-bromobutyrate was added. The reaction temperature rose to $80^{\circ} \mathrm{C}$, and reaction was carried out at this temperature for $80 \mathrm{~h}$. After the addition of $100 \mathrm{~mL}$ ice water into the reaction mixture, the water phase was extracted with carbon disulfide $(3 \times 50 \mathrm{~mL})$. The organic extracts were combined and washed with water, and dried over anhydrous magnesium sulfate. After removing the solvent by rotating evaporator, the product obtained was purified through column chromatography, and hexane/diethyl ether $(9-1 \mathrm{v} / \mathrm{v})$ was used as eluents. After the solvent was removed under reduced pressure, EPDTB was obtained as red oil ( $9.31 \mathrm{~g}$, yield $43.5 \%)$.

${ }^{1} \mathrm{H}$ NMR (500 MHz, $\left.\mathrm{CDCl}_{3}\right), \delta$ (TMS, ppm), 0.91(t $\left.3 \mathbf{H} \mathrm{CHCH}_{2} \boldsymbol{C H}_{3}\right), 1.25\left(\mathrm{t} 3 \mathbf{H} \mathrm{OCH}_{2} \boldsymbol{C H}_{3}\right), 1.56(\mathrm{~m}$ $2 \mathbf{H ~ C H C H} \mathrm{CH}_{2} \mathrm{CH}_{3}, 4.18\left(\mathrm{q} 2 \mathrm{H} \mathrm{OCH}_{2} \mathrm{CH}_{3}\right), 4.76(\mathrm{t} 1 \mathbf{H}$ $\left.\boldsymbol{C H} \mathrm{CH}_{2} \mathrm{CH}_{3}\right), 7.25(\mathrm{dd} 1 \mathbf{H} \mathbf{p}-\mathrm{ArH}), 7.35(\mathrm{dd} 2 \mathbf{H} \mathbf{~ m}-$ aromatic $\mathbf{H})$, 7.95(d $2 \mathbf{H ~ o - A r H})$.

\section{Polymerization}

The general procedures is as follows: Into a $5 \mathrm{~mL}$ polymerization tube with magnetic stirring bar, $1 \mathrm{~g}(6.2 \mathrm{mmol})$ of BMDO, $27.5 \mathrm{mg}(0.103 \mathrm{mmol})$ of EPDTB, $2.71 \mathrm{mg}(0.01 \mathrm{mmol})$ of DCP, and $1 \mathrm{~mL}$ THF were added. The mixture was degassed by three freezevacuum-thaw cycles. The tube was sealed under high vacuum, and then placed in an oil bath preheated at $120^{\circ} \mathrm{C}$ while stirring. After a prescribed time, the tube was put into ice water, cooled to room temperature and then opened. The polymer was obtained by pouring the polymer solution in THF into hexane while stirring; and the precipitate was collected by filtration, and dried at $40^{\circ} \mathrm{C}$ in vacuum overnight.

\section{Characterization of Polymers}

${ }^{1} \mathrm{H}$ and ${ }^{13} \mathrm{C}$ NMR spectra of the polymers obtained were recorded on a Bruker DMX-500 nuclear magnetic resonance spectrometer using $\mathrm{CDCl}_{3}$ as solvent, and tetramethylsilane (TMS) as internal reference. Molecular weight and polydispersity indexes were determined on a Waters $150 \mathrm{C}$ gel permeation chromatograph (GPC) equipped with Ultrastyragel columns $\left(500,10^{3}\right.$, $10^{4} \AA$ ) using monodisperse polystyrene as calibration standard. THF was used as an eluent at a flow rate of $1.0 \mathrm{~mL} \mathrm{~min}^{-1}$.

\section{RESULTS AND DISCUSSION}

\section{Free Radical Ring-Opening Polymerization}

The free radical ring-opening polymerization of BMDO was carried out at $120^{\circ} \mathrm{C}$ using EPDTB as chain transfer agent and DCP as initiator. The conditions and results are listed in Table I. The yield was measured by gravimetric method, and calculated according to eq 1 .

$$
\text { Yield }(\%)=\left[W_{\mathrm{p}} /\left(W_{\mathrm{I}}+W_{\mathrm{m}}\right)\right] \times 100 \%
$$

Where $W_{\mathrm{p}}$ is the weight of the isolated polymer and $W_{\mathrm{I}}$ and $W_{\mathrm{m}}$ are initial weights of initiator and monomer respectively.

As we know, BMDO underwent complete ringopening polymerization using conventional free radical initiator. $^{1}$ To clarify whether RAFT process of BMDO proceeds via complete ring-opening polymerization, ${ }^{1} \mathrm{H}$ and ${ }^{13} \mathrm{C}$ NMR spectra of the polymer obtained are shown in Figures 1 and 2. The signal at $\delta 5.06$ due to the identical methylene protons in the BMDO ring is split into two peaks at 5.10 and 2.91-2.96 (see Figure 1) corresponding to the two different methylene groups in the polyester. The former is the proton $\mathrm{i}$ between the ester oxygen and the benzene ring, while the latter is the proton $\mathrm{f}$ at the ortho position of the proton $\mathrm{i}$. The signal at 2.56-2.60 ppm corresponds the proton $\mathrm{d}$ of methylene 

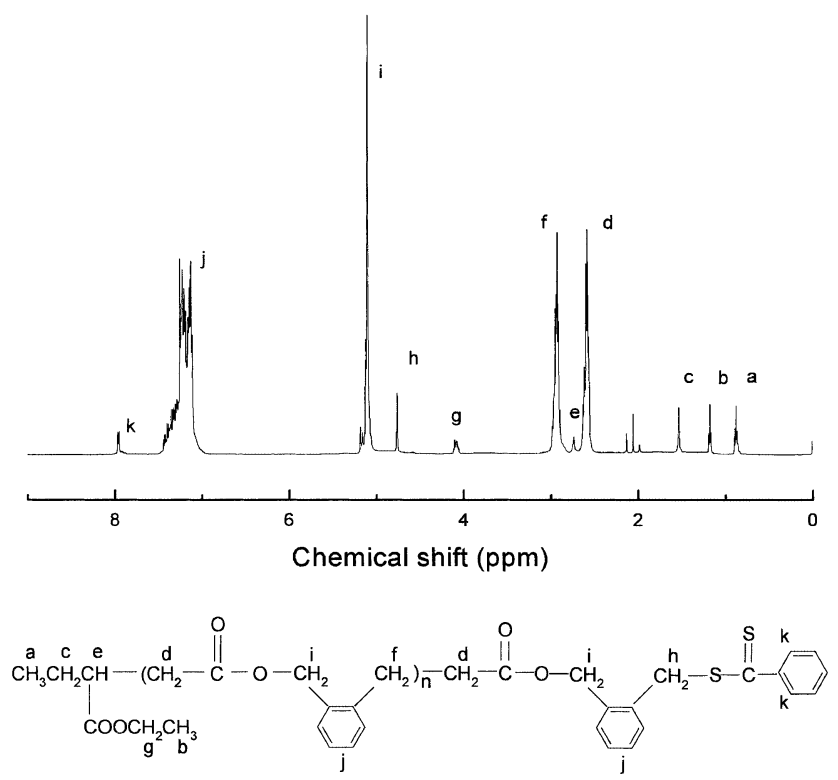

Figure 1. ${ }^{1} \mathrm{H}$ NMR spectra of PBMDO (No.2 in Table I).

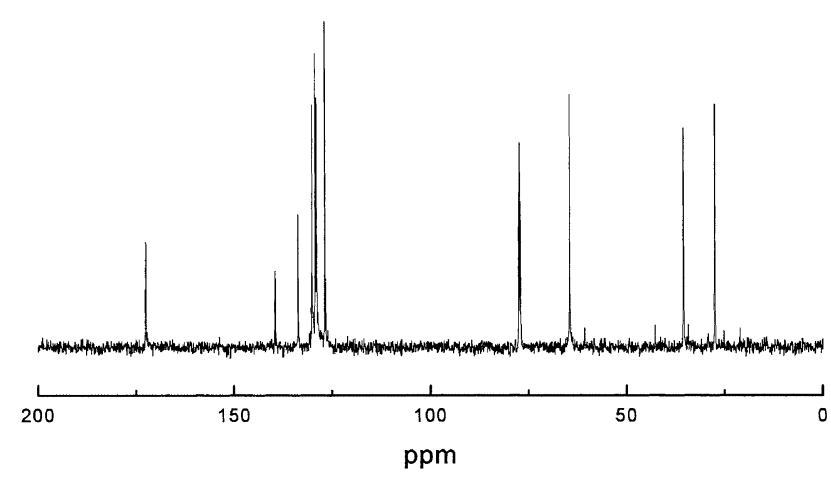

Figure 2. ${ }^{13} \mathrm{C}$ NMR spectra of PBMDO (No.2 in Table I).

group next to carboxylate group, which results from the ring-opening of 1,3-dioxepane ring. The integration ratio of $\mathrm{i}: \mathrm{f}: \mathrm{d}=1: 1: 1$ indicates that the polymer obtained is consisted of only ring-opened unit, so polyester was produced. Another evidences of complete ring-opening polymerization of BMDO lies in the facts that no acetal carbon signal at 95-120 ppm and ester carbon signal at 172.8 ppm appear in Figure 2.

Except the characteristic signals from PBMDO, several small peaks from transfer agent of EPDTB can be found in Figure 1. The signals at $\delta 0.91$ and 1.25 belong to the protons $\mathrm{a}$ and $\mathrm{b}$ of two methyl groaps in ethyl butyrate. The signals at $\delta 1.57$ and 4.18 are ascribed to methylene protons $\mathrm{c}$ adjacent methine group and $\mathrm{g}$ next to ester oxygen. The signal at $\delta 4.76$ of methine proton next to surfur in EPDTB is shifted to $\delta 2.74 \mathrm{ppm}$ (e) in Figure 1, and a new signal at $\delta 4.80$ correspondeol methylene protons next to surfur, indicating that ethyl butyrate and dithio benzoate are separately situated at the both end of the polymer chain.

Based on the above facts and general RAFT mech- anism, we proposed the free radical ring-opening polymerization mechanism of BMDO as shown in Scheme 2. Primary radical can initiate the polymerization of BMDO, or reacts with EPDTB to produce a new radical, butyrate radical, which initiates monomer to polymerize. Thus, we should observe a small amount of $\mathrm{Ph}\left(\mathrm{CH}_{3}\right)_{2} \mathrm{CO}$ groups stood at the end of polymer chain. Based on the ${ }^{1} \mathrm{H}$ NMR spectrum of DCP, the two methyl protons of $\mathrm{Ph}\left(\mathrm{CH}_{3}\right)_{3} \mathrm{CO}$ group appear at $\delta$ 1.60 , which is overlapped with methylene protons signal of butyrate group. This can be confirmed by the integration ratio $(1: 1.2)$ of the signal at $7.95 \mathrm{ppm}$ to that at $1.56 \mathrm{ppm}$, because the ratio of ortho aromatic protons to methylene protons of butyrate should be 2:2.

As we know, in RAFT process, the controlled behavior of the polymerization is maintained through the reversible addition fragmentation sequence between propagating chain radicals and dormant species with -SC(S)-Ph moiety. Comparison with conventional free radical ring-opening polymerization of BMDO initiated by DTBP at $120^{\circ} \mathrm{C}$ for $48 \mathrm{~h}$, polymerization in presence of EPDTB produced polyester in high yield, and with higher molecular weight as well as narrow polydispersity index (see Table I). The conventional radical polymerization of BMDO involves the propagation reaction via the reaction of chain radical with vinyl group of BMDO. It should be considered that the chain transfer reaction of chain radicals with methylene group of BMDO forms benzyl radical stabilized by benzene ring and oxygen. From filtration, we can obtain low molecular weight oligomers which may be an evidence of chain transfer reactions. As a result, after precipitation, the polyester with low yield and broad polydispersity was obtained. The fast reversible additionfragmentation reaction between propagating radicals and dormant polymer chain will reduce the concentration of propagating radicals, also lowered the possibility of chain transfer reaction. Since transfer reaction constant of chain radical with dithiobenzoate is larger than that with BMDO. This is the reason for the bettercontrolled natures of the polymerization, which will be discussed later.

Generally, free radical ring-opening polymerization requires higher reaction temperature. The polymerization of BMDO in the presence of EPDTB at $90^{\circ} \mathrm{C}$ produced polyester in very low yield (see No.6 in Table I). When the polymerization was performed in bulk, the polyester with broader molecular weight distribution $\left(M_{\mathrm{w}} / M_{\mathrm{n}}=1.53-1.76\right)$ were obtained. Linear relationship between molecular weight and conversion, and first order kinetic of polymerization were not observed. The possible reason may be explained as follows: after the polymerization was performed in bulk 


$$
\begin{aligned}
& \begin{array}{ccc}
\mathrm{Ph} & \mathrm{Ph} & \mathrm{Ph} \\
\left(\mathrm{CH}_{3}\right)_{2} \mathrm{C}-\mathrm{OO}-\mathrm{I} & \mathrm{C}\left(\mathrm{CH}_{3}\right)_{2} \longrightarrow 2 & \stackrel{1}{\mathrm{C}} \longrightarrow \\
\left(\mathrm{CH}_{3}\right)_{2} \mathrm{C}-\mathrm{O} .
\end{array}
\end{aligned}
$$

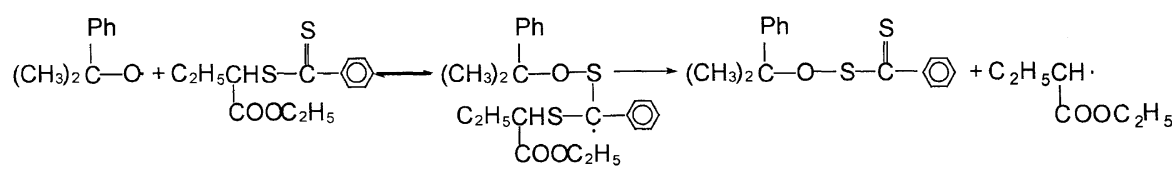<smiles>Cc1ccc(COC(COC(C)c2ccc(COCC(COC(C)c3ccccc3)OCc3ccccc3)cc2)COC(C)(C)c2ccccc2)cc1</smiles>

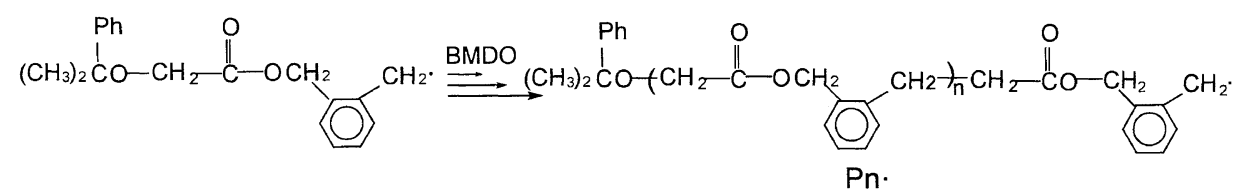
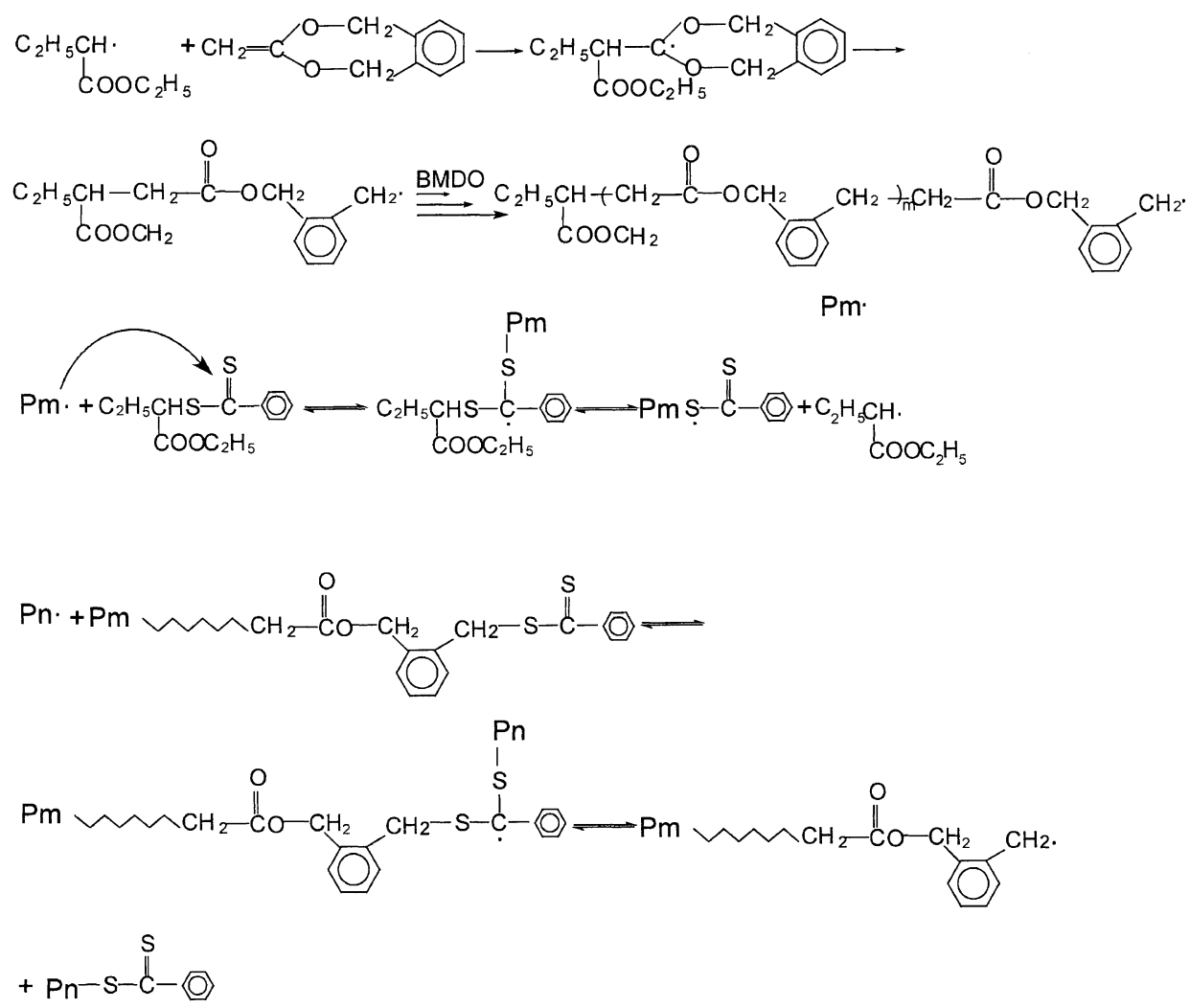

\section{Scheme 2.}

for a period of time, most of transfer agent EPDTB might be situated at the end of polymer chain, and the system viscosity increased, the possibility of encounter between PBMDO-SC(S)Ph and the chain radicals reduced. However, the chain transfer between the chain radical and BMDO did not affect very much. In these two competition reactions, the latter cannot be negligible. As a result, polymers with broad polydispersity index were obtained, and the increase of molecular weight with the conversion was not in accordance with a linear relationship.

\section{Controlled Characteristics of Polymerization}

To prove whether RAFT process of BMDO is a "living" polymerization, polymerizations in the different tubes were stopped at prescribed time intervals, and their conversions were measured. A plot of $\ln$ $\left[M_{0}\right] /[M]$ against polymerization time is shown in Figure 3. The straight line supports the constant concentration of propagating radicals during the polymerization until conversion reaches to $66 \%$, and termination of the radicals can be neglected.

Another evidence for the controlled polymerization is the linear relationship between $M_{\mathrm{n}, \mathrm{GPC}}$ and conversion as shown in Figure 4. Although monodisperse 
Table I. Conditions and experiment results of radical ring-opening polymerization of BMDO based on the RAFT method ${ }^{\mathrm{a}}$

\begin{tabular}{|c|c|c|c|c|c|c|c|c|}
\hline No. & Initiator $^{\mathrm{b}}$ & $\begin{array}{l}\text { Transfer } \\
\text { agent }^{c}\end{array}$ & Solvent & $\frac{\text { Time }}{\mathrm{h}}$ & $\frac{\text { Temp. }}{{ }^{\circ} \mathrm{C}}$ & $\frac{\text { Yield }}{\%}$ & $M_{\mathrm{n}, \mathrm{GPC}}{ }^{\mathrm{d}} / 10^{-3}$ & $M_{\mathrm{w}} / M_{\mathrm{n}}{ }^{\mathrm{d}}$ \\
\hline 1 & $\overline{\mathrm{DCP}}$ & EPDTB & THF & 10 & 120 & 16 & 1.8 & 1.32 \\
\hline 2 & DCP & EPDTB & THF & 22 & 120 & 33 & 3.6 & 1.34 \\
\hline 3 & DCP & EPDTB & THF & 32 & 120 & 48 & 5.0 & 1.30 \\
\hline 4 & DCP & EPDTB & THF & 42 & 120 & 60 & 6.4 & 1.29 \\
\hline 5 & DCP & EPDTB & THF & 52 & 120 & 66 & 7.0 & 1.27 \\
\hline 6 & DCP & EPDTB & THF & 32 & 90 & - & - & - \\
\hline 7 & DCP & EPDTB & - & 24 & 120 & 45 & 4.4 & 1.53 \\
\hline 8 & DCP & EPDTB & - & 45 & 120 & 50 & 5.3 & 1.57 \\
\hline 9 & DCP & EPDTB & - & 60 & 120 & 53 & 5.8 & 1.76 \\
\hline 10 & DTBP & - & - & 48 & 120 & 39 & 2.2 & 4.90 \\
\hline
\end{tabular}

${ }^{\text {a} P o l y m e r i z a t i o n ~ c o n d i t i o n s: ~ B M D O: ~} 1 \mathrm{~g}(6.2 \mathrm{mmol})$, EPDTB: $27.5 \mathrm{mg}(0.103 \mathrm{mmol})$, DCP: $2.71 \mathrm{mg}$ $(0.01 \mathrm{mmol})$, THF: $1 \mathrm{~mL}$. ${ }^{\mathrm{b}} \mathrm{DTBP}$ : di-tert-butyl peroxide, DCP: dicumy peroxide. ${ }^{\mathrm{c}} \mathrm{EPDTB}$ : 1 (ethoxycarbonyl)prop-1-yl-dithiobenzoate. ${ }^{\mathrm{d}}$ Estimated by GPC (polystyrene calibration).

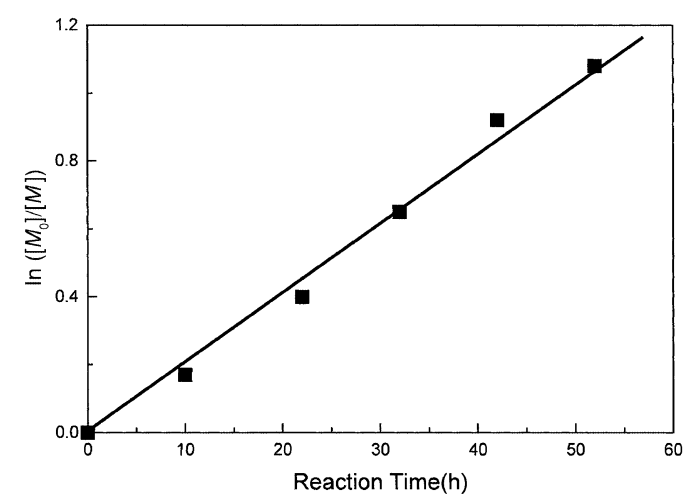

Figure 3. Relationship between $\ln \left(\left[M_{0}\right] /[M]\right)$ and polymerization time for solution polymerization of BMDO in THF at $120^{\circ} \mathrm{C}$.

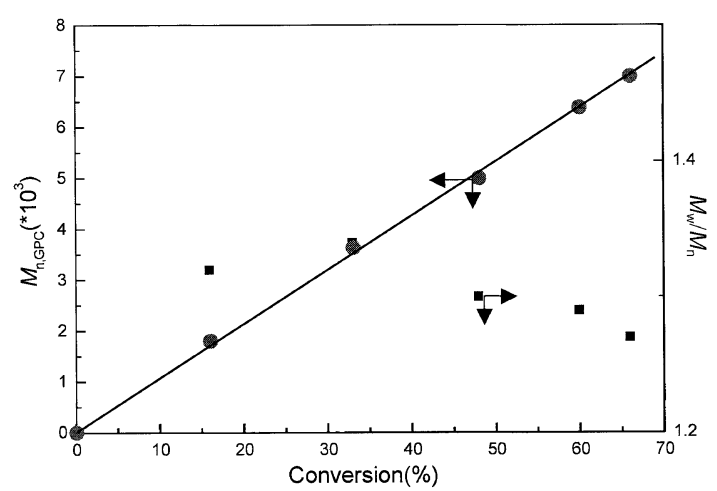

Figure 4. Dependence of $M_{\mathrm{n}, \mathrm{GPC}}$ and $M_{\mathrm{w}} / M_{\mathrm{n}}$ of conversion in the polymerization of BMDO in THF solution at $120^{\circ} \mathrm{C}$.

polystyrene standard was used in the calibration of molecular weight of PBMDO, $M_{\mathrm{n}, \mathrm{GPC}}$ does not affect the linear relation. Thus, the molecular weight of the polyester can be simply controlled by polymerization time. Further evidences for the controlled radical polymerization are the low polydispersity indexes (1.271.34) and the symmetric GPC curves as shown in Figure 5.

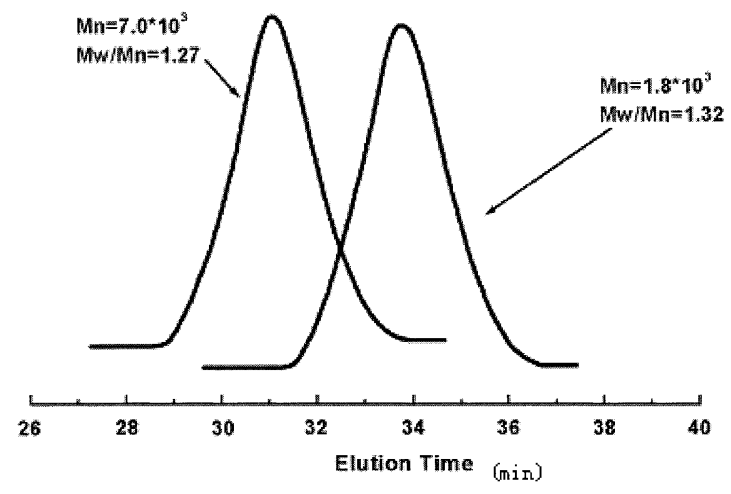

Figure 5. GPC curves of PBMDO of polymerization in THF solution at $120^{\circ} \mathrm{C}$ (No.1 and No.5 in Table I).

\section{CONCLUSION}

In the presence of EPDTB and DCP, BMDO underwent completely free radical ring-opening polymerization to produce polyester. The controlled radical polymerization of BMDO was supported by the following evidences: constant concentration of propagating chain radicals, linear relationship between molecular weight and conversion, lower polydispersity indexes $\left(M_{\mathrm{w}} / M_{\mathrm{n}}\right.$ $=1.27-1.34)$ and control of molecular weight by molar ratio of monomer to EPDTB or by polymerization time at molar ratio of EPDTB/DCP $>10$. Comparison with conventional radical polymerization of BMDO, this radical polymerization had high yield, and produced polyester with narrow molecular weight distribution, and well-controlled molecular weight.

Acknowledgment. This work is supported by the National Natural Science Foundation of China. (Grant 29774027). 


\section{REFERENCES}

1. W. J. Bailey, Z. Ni, and S.-R. Wu, Macromolecules, 15, 711 (1982).

2. W. J. Bailey, S.-R. Wu, and Z. Ni, Macromol. Chem., 183, 1913 (1982).

3. C. Y. Pan, Y. Wang, and W. J. Bailey, J. Polym. Sci., Part A: Polym. Chem., 26, 2737 (1988).

4. E. Klemm and T. Schalze, Acta Polymerica, 50, 1 (1999).

5. S. Jin and K. E. Gonsalves, Macromolecules, 30, 3104 (1997).

6. T. Okazaki, F. Sanda, and T. Endo, J. Polym. Sci., Part A: Polym. Chem., 34, 2059 (1996).

7. R. A. Evans and E. Rizzardo, Macromolecules, 29, 6983 (1996).

8. T. Okazaki, F. Sanda, and T. Endo, J. Polym. Sci., Part A: Polym. Chem., 35, 2487 (1997).

9. T. Koizumi, T. Ando, T. Kojimn, and T. Endo, Macromolecules, 31, 9096 (1998).

10. F. Sanda, Y. Takamasu, F. Hoshino, and T. Endo, J. Appl. Polym. Sci., 68, 497 (1998).

11. G. E. Roberts, M. L. Coote, J. P. A. Heuts, L. M. Morris, and T. P. Davis, Macromolecules, 32, 1332 (1999).

12. F. Sanda, T. Miyagawa, and T. Endo, Macromol. Chem. Phys., 200, 1089 (1999).

13. O. Moriya, T. Koizumi, T. Kodama, Y. Matsubara, S. Tsubaki, and T. Endo, J. Polym. Sci., Part A: Polym. Chem., 38, 3729 (2000).

14. R. A. Evans and E. Rizzardo, Macromolecules, 33, 6722 (2000).

15. W. J. Bailey, S.-R. Wu, and Z. Ni, J. Macromol. Sci. Chem., A 18, 973 (1982).

16. W. J. Bailey, Macromol. Chem. Suppl., 13, 171 (1985).

17. W. J. Bailey, J.-M. Gu, Y.-N. Lin, Z.-F. Zheng, and L. L. Zou, Makromol. Chem. Macromol. Symp., 42/43, 195 (1991).
18. W. J. Bailey, C.-Y. Pan, and S.-C. Chen, Makromol. Chem. Makromol. Symp., 6, 81 (1986).

19. J. P. He, L. Li, and Y. L. Yang, Macromol. Theory Simul., 9, 463 (2000).

20. C. Farcet, M. Lansalot, B. Charleux, R. Pirri, and J. P. Vairon, Macromolecules, 33, 8559 (2000).

21. X. Z. Ding, A. Fischer, A. Brembilla, and P. Lochon, J. Polym. Sci., Part A: Polym. Chem., 38, 3067 (2000).

22. M. Nowakowska, S. Zapotoczny, and A. Karewicz, Macromolecules, 33, 7345 (2000).

23. S. Kobatake, H. J. Harwood, R. P. Quirk, and D. B. Priddy, J. Polym. Sci., Part A: Polym. Chem., 36, 2555 (1998).

24. J.S. Wang and K. Matyjaszewski, Macromolecules, 28, 7901 (1995).

25. M. Kato, M. Kamigaito, M. Sawamoto, and T. Higashimura, Macromolecules, 28, 1721 (1995).

26. V. Percec, H. S. Kim, and B. Barbonl, Macromolecules, 30, 6702 (1997).

27. Y. K. Chong, T. P. T. Le, G. Moad, E. Rizzardo, and S. H. Thang, Macromolecules, 32, 2071 (1999).

28. J. Chiefari, Y. K. Chong, F. Ercole, J. Kristine, J. Jeffery, T. P. T. Le, R. T. A. Mayaduwne, G. F. Meijs, G. Moad, E. Rizzardo, and S.H. Thang, Macromolecules, 31, 5559 (1998).

29. K. Matyjaszewski, in "Controlled Radical Polymerization" K. Matyjaszewski, Ed., ACS Symposium Series, American Chemical Society, Washington, D. C. 1998, vol. 685, pp 911.

30. Y. Wei, E. J. Connors, X. Jia, and C. Wang, J. Polym. Sci., Part A: Polym. Chem., 36, 761 (1998).

31. X. Jia, M. Li, S. Han, C. Wang, and Y. Wei, Mater. Lett., 31, 137 (1997).

32. C.-Y. Pan and X.-D. Lou, Macromol. Chem. Phys., 201, 1115 (2000).

33. G.-Y. Yuan, C.-Y. Pan, and B.-Z. Tang, Macromolecules, 34, 211 (2001). 